\title{
Mean Velocity Charateristics in the Far Wake behind a GAW(2) Airfoil
}

\author{
N. Subaschandar \\ Department of Mathematics and Computational Sciences, BIUST, Palapye, Botswana
}

\begin{abstract}
The two-dimensional asymmetric far wake behind a GAW(2) airfoil section is studied at low speeds. Mean velocity measurements have been made and analysed in the far wake, where initial asymmetric wake has become symmetric with respect to the minimum velocity line. Governing equations have been solved with appropriate boundary conditions in the far wake. Self-similarity solutions have been obtained for the governing equations for the mean velocity distributions. Results of self-similarity analysis have been compared with experimental data.
\end{abstract}

\section{Introduction}

Turbulent wake region is the region of flow behind the trailing edge of a body where the upstream boundary layers merge into one single shear layer. The far wake region is at a large distance from the trailing edge where the flow is less influenced by the initial conditions at the trailing edge. The evaluation and understanding of flow in the far wake is important as the flow in this region is solely dependent on the gross parameters like total drag of the body. The aim of the present study is to analyse and understand far wake flow developing from initial asymmetric trailing edge conditions. The GAW(2) airfoil which is a $13 \%$ thickness General Aviation Wing was chosen for this study because of its strong relevance in many applications. Many of the studies carried out in the past were concentrated in the symmetric far wake flows[1-5]. Self-similarity solutions have been obtained in the symmetric far wake in such flows. The far wake flow, which is developing from initial asymmetric conditions at the trailing edge, has not been studied well. The present research was started with an aim to describe far wake, with initial asymmetric conditions, in terms of self-similarity solutions for governing equations. The three-dimensional asymmetric turbulent near wake behind a swept wing with GAW(2) airfoil cross section has been studied extensively[6]. Their studies showed that the turbulent wake which was asymmetric near the trailing edge exhibited symmetry by about a distance of 60 trailing edge momentum thicknesses downstream of the trailing edge[6]. With this in mind, our focus was concentrated in the wake region beyond a distance of 200 trailing edge momentum thicknesses downstream of the trailing edge so that symmetry has been reached in the region of our interest.

\section{Experimental Setup}

The experiments were conducted in the $300 \times 1500 \mathrm{~mm}$ boundary layer wind tunnel of the National Aerospace Laboratories, Bangalore. The GAW(2) airfoil model, having a chord length of $600 \mathrm{~mm}$ and a span of $300 \mathrm{~mm}$, was mounted vertically in the test section. The experiments were carried out at a freestream velocity of $30 \mathrm{~m} / \mathrm{s}$, providing a chord Reynold number of $1 \times 10^{6}$. Figure 1 shows the schematic of the experimental setup. Mean velocity measurements were carried out in the wake in the distance range of 1-800 momentum thicknesses behind the trailing edge. The mean velocity profiles are presented at three streamwise stations furthest from the trailing edge so as to avoid the uncertainty regarding the symmetry of the flow.

\section{Similarity Analysis}

In this section, an attempt is made to extend the selfsimilarity analysis which is valid for symmetric twodimensional far wake to the far wake flow evolving from initial asymmetric trailing edge conditions. In this section far wake flow is analysed using continuity and momentum equations. Most of the analysis follows the steps of two-dimensional far wake analysis given in[4].

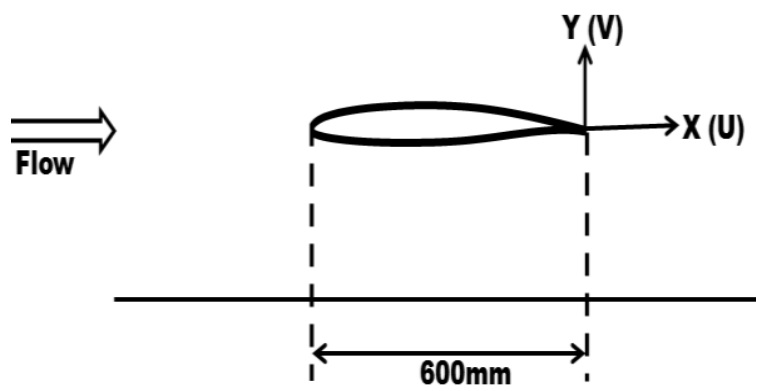

Figure 1 Schematic of the experimental setup 
With the notations shown in Figure 1, the equations for the far wake flow are

$$
\begin{gathered}
\frac{\partial \mathrm{U}}{\partial \mathrm{X}}+\frac{\partial \mathrm{V}}{\partial \mathrm{Y}}=0 \\
\mathrm{U} \frac{\partial \mathrm{U}}{\partial \mathrm{X}}+\mathrm{V} \frac{\partial \mathrm{U}}{\partial \mathrm{Y}}=\frac{1}{\rho} \frac{\partial \tau}{\partial \mathrm{Y}}
\end{gathered}
$$

The freestream velocity outside the wake is assumed to be constant $\left(\mathbf{U}_{\infty}\right)$ and $\mathbf{x}=\boldsymbol{\mu} \frac{\boldsymbol{\partial U}}{\boldsymbol{\partial y}}-\boldsymbol{\rho} \overline{\mathbf{u v}}$ is the total shear stress. We seek a similarity solution of the form

$$
\tau=\rho W_{0}^{2} g(\eta), W=W_{0} f(\eta)
$$

where $\mathrm{W}=\mathrm{U}_{\infty}-\mathrm{U}$ and $\eta=Y / B$ and $\mathrm{W}_{0}$ and $\mathrm{B}$ are the local velocity and length scales, respectively. Eqs 1 and 2 reduce to

$$
\begin{aligned}
& {\left[\frac{\mathrm{U}_{\infty} \mathrm{B}^{\prime}}{\mathrm{W}_{0}}-\underline{\mathrm{B}^{\prime} \mathrm{f}}\right] \eta \mathrm{f}^{\prime}-\left[\frac{\mathrm{U}_{\infty} \mathrm{W}_{0}^{\prime}}{\mathrm{W}_{0}^{2}} \mathrm{~B}-\frac{\mathrm{W}_{0}^{\prime} \mathrm{B}}{\mathrm{W}_{0}} \mathrm{f}\right] \mathrm{f}-} \\
& \mathrm{f}^{\prime} \int\left\{\frac{\mathrm{w}_{0}^{\prime} \mathrm{B}}{\mathrm{W}_{0}} \mathrm{f}-\mathrm{B}^{\prime} \eta \mathrm{f}^{\prime}\right\} \mathrm{d} \eta-\mathrm{g}^{\prime}=0
\end{aligned}
$$

At large distances from the body, the velocity defect is small, i.e $\mathrm{W}_{0}<<\mathrm{U}_{\infty}$, and the underscored terms are of second order or smaller. Hence

$$
\frac{\left(\mathrm{U}_{\infty 0} \mathrm{~B}\right)^{r}}{w_{0}} \eta \mathrm{f}^{\prime}-\frac{\mathrm{B}\left(\mathrm{U}_{\infty 0} w_{0}\right)^{r}}{w_{0}^{2}} \mathrm{f}-\mathrm{g}^{\prime}=0
$$

If this asymptotic wake is self-preserving, then Eq. 5 leads to the following well-known result for a far wake with a nonzero momentum thickness[7,8]

$$
\mathrm{B} \sim \mathrm{X}^{1 / 2} \text { and } \mathrm{W}_{0} \sim \mathrm{X}^{-1 / 2}
$$

These half-power growth and decay laws can therefore be used to measure the approach of the mean flow to asymptotic conditions.

In order to evaluate the constants in Eq. 6 and determine the velocity profile $f(\eta)$, it is necessary to invoke a turbulence closure model. Thus, for example, introduction of a constant eddy-viscosity, $\mathbf{v}_{\mathbf{T}}$, viz

$$
\frac{\tau}{\rho}=v_{T} \frac{\partial U}{\partial Y}=v_{T} \frac{W_{0}}{B} f^{\prime} \text { or } g=\frac{-v_{T}}{W_{0} B} f^{\prime} .
$$

reduces Eq.5 to

$$
\frac{v_{\mathrm{T}}}{w_{0} \mathrm{~B}} \mathrm{f}^{\prime \prime}+\left(\frac{\mathrm{U}_{\infty} \mathrm{B} r}{w_{0}}\right) \eta \mathrm{f}^{\prime}-\left(\frac{\mathrm{U}_{\infty} \mathrm{B} w_{0}^{\prime}}{w_{0}^{2}}\right) \mathrm{f}=0
$$

which is identical with that for laminar flow except that $\mathbf{v}$ has to be replaced by $\mathbf{v}_{\mathbf{T}}$ [7]. With the appropriate boundary conditions, the solution of Eq. 7 is

$$
\begin{aligned}
& \mathbf{f}=\frac{w}{w_{0}}=\exp \left(-4 \eta^{2} \ln 2\right) . \\
& B=4 \sqrt{\ln 2}\left(\frac{v_{\mathrm{T}} \mathrm{X}}{\mathrm{U}_{\infty}}\right)^{1 / 2} .
\end{aligned}
$$

$$
\frac{w_{0}}{U_{\infty}}=\frac{1}{\sqrt{\pi}}\left(\frac{U_{\infty} C_{\mathrm{d}} d}{v_{T}}\right)^{1 / 2}\left(\frac{C_{\mathrm{d}} \mathrm{d}}{\mathrm{X}}\right)^{1 / 2}
$$

where $\mathbf{C}_{\mathbf{d}}=\frac{\mathbf{D}}{\left(\frac{1}{2}\right) \boldsymbol{\rho} \mathbf{U}_{\infty}^{2} \mathbf{d}}$ is the drag coefficient and $\mathrm{d}$ is a characteristic length of the body. The overall momentum shows that $\mathrm{C}_{\mathrm{d}} \mathrm{d}=2 \theta$ where

$$
\theta=\int_{-\infty}^{\infty} \frac{\mathrm{U}}{\mathrm{U}_{\infty}}\left(1-\frac{\mathrm{U}}{\mathrm{U}_{\infty}}\right) \mathrm{dY} \text {. }
$$

is the constant, asymptotic momentum thickness of the wake. Using $\boldsymbol{\theta}$ as a length scale, equations (9) and (10) become

$$
\begin{aligned}
& \left(\frac{\mathrm{B}}{\mathrm{\theta}}\right)^{2}=16\left(\frac{\mathrm{v}_{\mathrm{I}}}{\mathrm{U}_{\infty} \theta}\right)\left(\frac{\mathrm{X}}{\theta}\right) \ln (2) . \\
& \left(\frac{\mathrm{U}_{\infty}}{w_{0}}\right)^{2}=4 \pi\left(\frac{v_{\mathrm{T}}}{\mathrm{U}_{\infty} \theta}\right)\left(\frac{\mathrm{X}}{\theta}\right) .
\end{aligned}
$$

From an examination of some early results from the wake of cylinders, Schlichting deduced a value of 0.0444 for $\frac{\mathbf{v}_{\mathrm{T}}}{\mathbf{v}_{\omega}}[7]$. However, Townsend quotes a value of $0.032[8]$, which is identical with the average value deduced from the asymptotic wake data[5]. A value of 0.032 was found to be suitable in the present analysis also. It should be noted that the constants in the above results are derived on the basis of a constant eddy-viscosity model, which is not entirely realistic in view of the marked intermittency of the flow over a large part of the wake. 


\section{Results and Discussion}

In this section, results of the above analysis are compared with the data measured in the asymmetric wake behind a GAW(2) airfoil. The appropriate value of $\boldsymbol{\theta}$ was determined from the data of the most downstream station in order to avoid any uncertainties occurring from the measurements near the trailing edge. Figure 2 shows measured mean velocity profiles in the far wake flow at several streamwise stations. Since it was observed that the velocity profiles had reached symmetry only one half of the profiles are shown in the figure. Also, shown in the figure is the self-similar exponential curve given in the Eq. 8. It can be seen from this figure that the measured data are in good agreement with the exponential asymptotic profile except for some small departures near the outer edge of the wake.

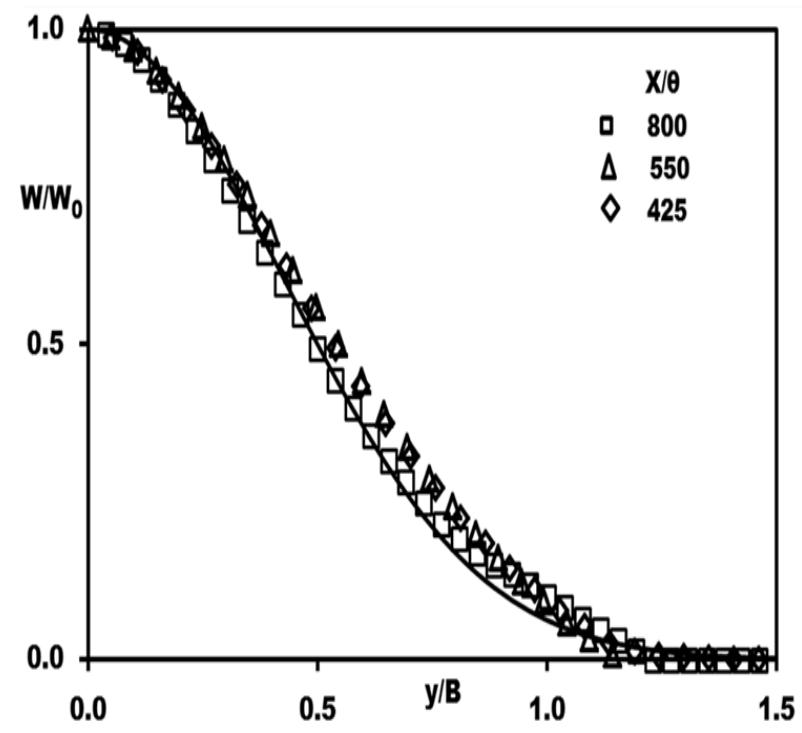

Figure 2 Mean velocity profiles in similarity variables.

Figure 3 shows the variation of wake half thickness in the far wake. This figure also shows the relationship for wake half thickness as given by the Eqs.11 with $\frac{\mathbf{y}_{\mathrm{T}}}{\mathbf{U}_{\infty} \mathrm{\theta}}=0,032$. From this figure it is clearly seen that the wake half thickness does not follow the asymptotic power laws with the prescribed constant value of eddy-viscosity until about $\mathrm{X} / \theta=300$ and after that the agreement between the measured data and the analytical results is reasonably good.

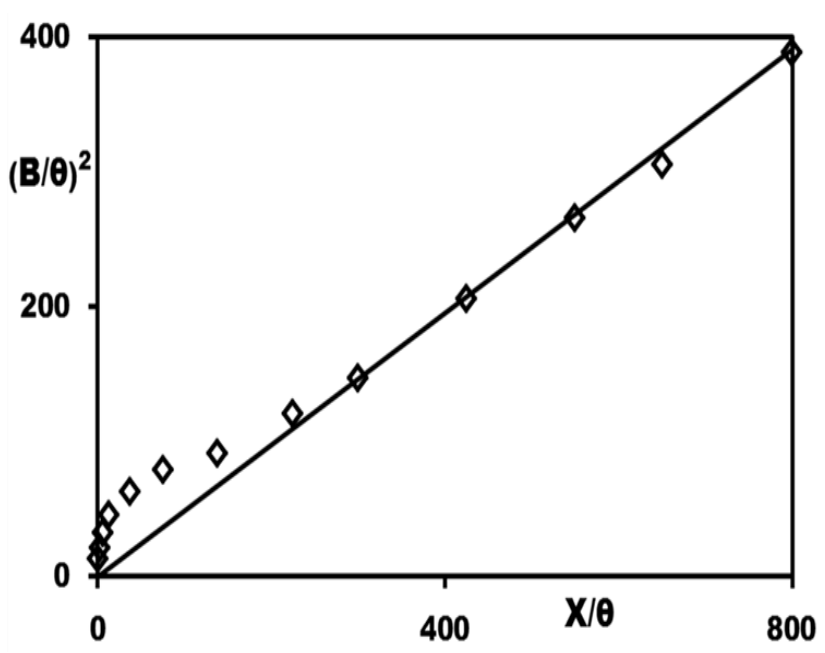

Figure 3 Streamwise development of wake half-thickness

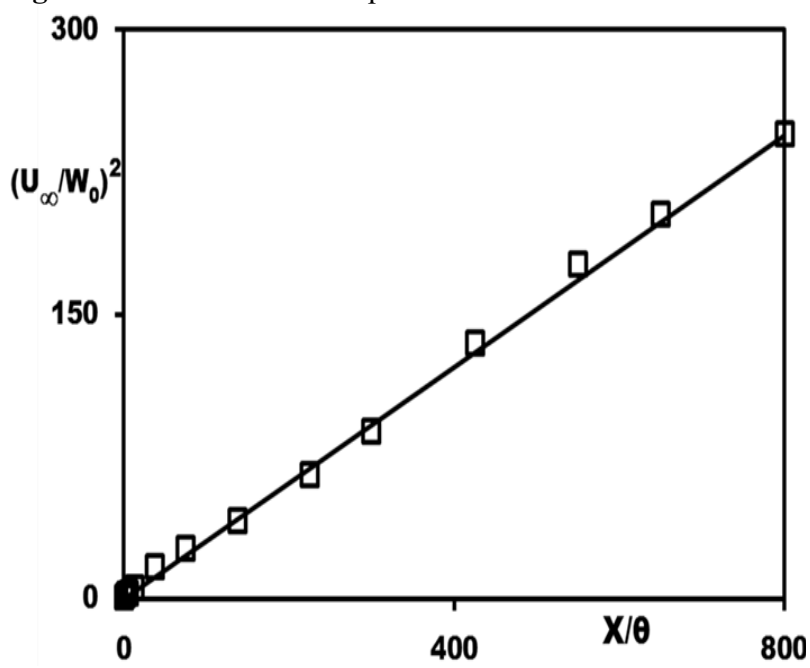

Figure 4 Streamwise development of inverse of maximum velocity defect

Figure 4 shows the variation of inverse of maximum velocity defect in the far wake. This figure also shows the relationship for inverse of maximum velocity defect as given by the Eq. 12 with $\frac{\mathbf{v}_{\mathrm{T}}}{\mathbf{v}_{\infty 8} \mathbf{\theta}}=0.032$. From this figure it is clearly seen that the inverse of the maximum velocity defect does not follow the asymptotic power law with the prescribed constant value of eddy-viscosity until about $\mathrm{X} / \theta=300$ and after that the agreement between the measured data and the analytical results is reasonably good.

In summary, the experimental data in the turbulent wake behind a GAW(2) airfoil indicate that the velocity profiles have become symmetric in the far wake. The experimental data in the turbulent far wake behind the GAW(2) airfoil indicate that the wake becomes independent of the initial conditions and approaches as asymptotic state in mean velocity at around $X / \theta=300$.

\section{Conclusions}

Mean velocity measurements have been carried out in the far wake flow region behind a GAW(2) airfoil. It is shown that the self-similarity analysis of a symmetric two-dimensional far wake flow can be extended to the far 
wake flow of an initially asymmetric wake flow. The results of self-similarity analysis agree well with experimentally measured mean velocity data in the far wake. The wake half-thickness and inverse of maximum velocity defect agree well with the experimental data in the far wake and shown to follow the half power laws derived in the self-similarity analysis.

\section{Acknowledgements}

Author acknowledges the support received from the Department of Mathematics and computational Sciences and BIUST authorities in carrying out this research.

\section{References}

1. R. Chevray and L. G. Kovasznay, AIAA J., 7(8), 1641, (1969).

2. P. J. Pot, NLR TR 79063, National Aerospace Laboratory NLR, Netherlands, (1979).

3. J. Andreopoulos and P. Bradshaw, J. of Fluid Mech., 100, 639, (1980).

4. B. R. Ramaprian, V. C. Patel, and M. S. Sastry, Iowa Institute of Hydraulic Research Report 231, University of Iowa, Iowa City USA, (1981).

5. R. Narasimha and A. Prabhu,, J. of Fluid Mech., 54, 1 (1972).

6. N. Subaschandar and A. Prabhu, J. of Aircraft, 39, No 2, 290 (2002)

7. H. Schlichting, Boundary Layer Theory, McGrawHill Book Co., New York, (1968)

8. A. A. Townsend, Turbulent Shear Flow, Oxford University Press, G. B., (1956). 